\title{
HOW COVID-19 PATIENT NARRATIVES CONCERNING REINFECTION MIRROR THEIR MENTAL HEALTH: A CASE SERIES
}

\author{
Yu Deng ${ }^{1}$, Li Wang ${ }^{2}$, Jixue Yang ${ }^{3}$, Luxue Xie ${ }^{3}$ \& Yaokai Chen ${ }^{2}$ \\ ${ }^{I}$ College of Language Intelligence, Sichuan International Studies University, Chongqing, China \\ ${ }^{2}$ Division of Infection Diseases, Chongqing Public Health Medical Center, Chongqing, China \\ ${ }^{3}$ School of School of English Studies, Sichuan International Studies University, Chongqing, China
}

Yu Deng \& Li Wang contributed equally to this work

received: 18.2.2021;

revised: 5.3.2021;

accepted: 8.3.2021

\section{INTRODUCTION}

COVID-19 has resulted in an increasing threat to the physical and mental health of humans. A growing body of literature concerning mental health and COVID-19 show that the ongoing pandemic has led to increased psychiatric disorders such as post-traumatic stress, depressive and anxiety disorders, as well as grief-related symptoms (Bao et al. 2020, Dong et al. 2020, Guessoum et al. 2020, Islam et al. 2020, Rajkumar 2020). Studies on the mental health of patients with COVID-19 have indicated that patients experience boredom, loneliness, anger, insomnia, anxiety, depression, and distress (Bao et al. 2020, Chen et al. 2020, Duan \& Zhu 2020, Luo et al. 2020; Muruganandam et al. 2020, Wang et al. 2020, Xiang et al. 2020).

While many hospitalized COVID-19 patients recovered and were discharged to self-quarantine, some patients were re-tested positive for COVID-19 post discharge (Mei et al. 2020). As retest-positivity of COVID-19 could be a major aggravating factor in the current pandemic, there has been an increase in reports of potential COVID-19 retest-positivity following initial recovery (Arafkas et al. 2021, Bo et al. 2020, Bongiovanni 2021, Iwasaki 2020, Lafaie et al. 2020, Lee et al. 2020, Mei et al. 2020, Parry 2020, Prado-Vivar et al. 2020, Ravioli et al. 2020). What these studies have in common is that they principally concentrate on physical symptoms of patients with a positive retest. Despite numerous studies regarding mental health of COVID-19 patients' initial infection, there appears to be little published work that has seriously addressed the dynamic mental difficulties of COVID-19 patients with two or more separate retest-positive results. There is no doubt that mental health issues pose major challenges in patients with a positive retest. For instance, recent findings have shown that retest-positive patients had increased suicidal ideation (Mei et al. 2020).

To date, while a considerable number of surveys have been conducted to address COVID-19 patients' mental health problems, it seems that narrative data concerning the dynamic psychological experiences of recurrent COVID-19 patients are currently underestimated (e.g., Sahoo et al. 2020a, 2020b). This study reports on a group of six COVID-19 patients who narrated their experiences of two distinct hospital admissions due to positivity for COVID-19 through in-depth semi-structural interviews. We probe patients' mental health problems during the periods of both recovery and recurrence, and offer insights into psychological interventions for COVID-19 patients at different stages.

\section{METHOD}

Six COVID-19 patients who retested positive after initial recovery attended the semi-structural interview by cellphone, as paid volunteers. Table 1 shows the participants' demographic information. Written informed consent was obtained from these participants before the interview. The study was conducted according to the guidelines of the Declaration of Helsinki, and approved by the Ethics Committee of Chongqing Public Health Medical Center.

Table 1. Demographic Information of Participants

\begin{tabular}{ccccccccc}
\hline $\begin{array}{c}\text { Subject } \\
\text { ID }\end{array}$ & Gender & Nationality & Age & $\begin{array}{c}\text { Marital } \\
\text { Status }\end{array}$ & Occupation & $\begin{array}{c}\text { Time in } \\
\text { Hospital }\end{array}$ & $\begin{array}{c}\text { Infection } \\
\text { Locality }\end{array}$ & $\begin{array}{c}\text { Severity } \\
\text { Level }\end{array}$ \\
\hline 1 & Female & Chinese & 35 & Y & Businesswoman & 47 days & Chongqing & Mild \\
2 & Female & Chinese & 47 & Y & Housewife & 60 days & Chongqing & Severe \\
3 & Male & Chinese & 30 & Y & Worker & 18 days & Singapore & Mild \\
4 & Male & Chinese & 31 & N & Businessman & 15 days & Wuhan & Mild \\
5 & Male & Chinese & 49 & Y & Farmer & 42 days & Chongqing & Moderate \\
6 & Male & Chinese & 34 & Y & Unemployed & 54 days & Chongqing & Mild \\
\hline
\end{tabular}


During the interview, the six retest-positive patients narrated their experiences of frustration and feelings concerning COVID-19 during the stages of recovery and recurrence. The interview questions included how they were infected and re-tested positive for COVID-19, what they did before, during, and after hospitalization, how their work and daily life was affected in the first and second admission, what gave them comfort, how they felt while admitted in hospital, and how they dealt with social relationships after discharge. Each interview lasted about 40 minutes. The interviews were recorded and transcribed for analysis.

\section{CASE PRESENTATIONS}

\section{$1^{\text {st }}$ Case: "Where is the end of COVID-19?"}

The 35-year-old woman (subject 1) was admitted to hospital again after a positive retest result one day after her discharge from hospital after initial infection. When she was diagnosed with the infection of COVID-19 for the first time, she believed that "COVID-19 was not a serious disease" due to her trust in the expertise of doctors. However, this optimistic belief turned to irritation and anxiety when she tested positive again after recovery. She did not know "where is the end of the disease", and felt that "she was caught in an endless loop of 14-day-quarantines". She considered herself to be "sentenced to imprisonment for life" due to the unavailability of effective therapeutic medication for COVID-19 currently. Her spirit was "broken down", and she could not fall asleep without hypnotics. Crying and screaming in the ward, she "felt no different from a psycho". Consumed by extreme confusion and stress, she even dreamed about her dead mother visiting her and chatting with her. She was too desperate to talk with others and tried to isolate herself. Psychological guidance in the hospital had little effect on her due to her mistrust of everyone, including the doctors and nurses who took care of her. She constantly inquired about the treatment plan and indulged in taking medicine, insisting that "she was helpless if the doctor did not prescribe medicine". Fortunately, fellow patients in the same ward as her encouraged her a lot. According to her narration, "the words of her family were powerless" and "failed to reach the deep side of her heart" given that they could not understand her confinement in the hospital. Only those who suffered from the same experience as her could relieve her concerns and "touch upon the deep side of her heart".

When she returned home after recovery, she still considered herself as a patient who should be quarantined in the hospital or somewhere far away from her family. She was scared that "her brain would break down" as a result of her constant imagination about her misfortune. Running around the house in the middle of the night plagued by insomnia, she "had the impulse to commit suicide". She was reluctant to go out because she was afraid of being isolated in the hospital again. It is noteworthy that, according to her report, her life had not returned to normal and she felt that she was being supervised by others due to the frequent calls to her in order to gather research data.

\section{$2^{\text {nd }}$ Case: "My kidneys were going to jump out of my body"}

The 47-year-old woman (subject 2) was initially hospitalized after she tested positive for COVID-19. Three sisters in her extended family were infected, amongst whom her older sister showed severe symptoms. During her first hospitalization, she was optimistic as she only developed cough as a symptom. As expected, she reached the clinical threshold for discharge from the hospital in a week. Afterwards, she actively donated plasma in order to contribute to research on fighting COVID-19. However, she tested positive for COVID-19 again on the 16th day of her hotel quarantine. She demonstrated severe symptoms of cough and expiratory dyspnea. The retest-positivity "aggravated her emotional state". Furthermore, spread of the news regarding her re-positivity resulted in her becoming "mentally vulnerable". Discrimination from neighbors, and rumors such as "I wonder how many people may be endangered by her plasma" increased her grief. Compared with her first hospitalization when she usually danced, and had optimistic video chats with her sisters, she became agitated and reluctant to answer calls during the second hospitalization, and felt that "constant inquiries about the re-positivity from relatives and friends irritated her". She avoided contacting her elder sister who was in a serious condition, lying to her that she stayed at her friend's home. Several positive tests for COVID-19 at the hospital led her to a state of despair.

Receiving treatment at the hospital for nearly two months, she was cured and sent to a hotel for quarantine again. According to regulations, three consecutive negative results of the weekly nucleic acid test were required for release from hotel quarantine. She tested negative in the first two weeks, but retested positive in the third week. She refused to be admitted to hospital again, and was unwilling to take medicine due to the severe side-effects of the drugs. She repeatedly stated that "her fingers had become numb" and "her kidneys were going to jump out of her body". After being released from quarantine, discrimination and shunning by her neighbors persisted, which "made her feel cold".

\section{$3^{\text {rd }}$ Case: "I am not a monster spreading virus"}

As a decoration worker in Singapore, the 30-yearold man (subject 3 ) lived in a dormitory shared by more than 200 people, 90 percent of whom were infected by COVID-19. When he tested positive for COVID-19 he became extremely anxious and frightened by his elevated heart rate. Recalling what he had experienced, he considered the dormitory as "a room filled with the 
virus". During hospitalization, he persistently inquired online from doctors in China about possible treatments for COVID-19.

After being discharged from hospital, he returned to China immediately. Unfortunately, he was tested positive for COVID-19 again on the 14th day of hotel quarantine, which alarmed him tremendously. Reading about cases of reinfection online increased his anxiety, worry, and sense of panic. Since he began his quarantine immediately after returning to China, he could not pay all of the required quarantine fee. He felt embarrassed to seek help from his family because "he was afraid of mentioning his re-positivity to the family, in particular to his mother who had heart disease". Without support from his family, he experienced extraordinary loneliness in hospital quarantine. Even after he was discharged, "the memory of lying on the bed with loneliness, panic, pressure, and helplessness lingered". He was reluctant to share his experiences after retesting positive with his friends and relatives, in the sense that he thought that they may consider him to be "a monster spreading virus". At the beginning of his hospitalization, he only lost his sense of taste and smell, and had no other symptoms. Following this, he developed fever. The symptomatic reoccurrence disturbed him so much that he instructed his nephew to disinfect all the cash that he had brought back from Singapore, and to forward it to his family in the event of his death. Furthermore, he slept very lightly and frequently woke up at midnight with stress. Reflecting on his life, he regretted wasting time in the past and was determined to create a better life for his family. In summation, he was in a state of panic, anxiety, and loneliness in hospital quarantine. Thankfully, medical staff at the hospital took good care of him and this "warmed his heart" in the Chinese hospital.

\section{$4^{\text {th }}$ Case: "Re-positivity is a knot in my heart"}

The 31-year-old man (subject 4) was confirmed to be infected by COVID-19 and admitted to hospital about four to five days after he returned home from Wuhan by train. Learning that he was infected, he felt very flustered and disappointed. The news about the outbreak in Wuhan bothered him, but he was optimistic that he would recover soon. He got along well with his fellow patients in the isolation ward by playing games and chatting together. None of them had severe symptoms. He was quite tranquil and open-minded at the beginning of his hospitalization. However, he felt like he was "walking through the gates of hell" when he witnessed a fellow patient who had mild symptoms worsening rapidly, and who was sent to the intensive care unit within one day. Fortuitously, he recovered and was discharged from the hospital soon after. Unfortunately, he was tested positive again on the last day of his 2-week hotel quarantine. After being hospitalized for the second time, he could not help "swearing and shouting with extreme annoyance". A deep sense of mental suffering rose steeply "in a geometric pro- gression". He was very prone to losing his temper and complained to the medical staff about the food. He soon calmed down and apologized for his rude behavior. Instead of worrying about his future, he focused on complying with the treatment. He believed that "he was in a gray area with darkness on one side and brightness on the other". Overall, he reported an attitude of optimism throughout the hospitalization. Nevertheless, the re-positivity of COVID-19 still affected his life after he recovered, as some people required him to keep a distance away from them. The thought of being positive again was "a knot in his heart", in that he worried about losing his job and about facing financial pressure in the near future.

\section{$5^{\text {th }}$ Case: "Why people discriminate me after my recovery"}

The 49-year-old farmer (subject 5) was admitted to the COVID-19 ward after testing positive for the infection. As far he recollected, he was infected by his wife who had met people from Hubei Province. During the hospitalization, he received treatment in isolation. He had flu symptoms of slight cough, fever, and fatigue. He was extremely worried about the fruit sales in his farm, but his anxiety was alleviated soon after since the local government assisted in fruit sales. 12 days later, he was discharged from the hospital as he had tested negative. He adjusted his mood through outside activities such as fishing and driving. Leaving hospital made him so excited that "he wanted to jump up" and was grateful that "he was liberated at last". Unfortunately, he retested positive for COVID-19. He felt depressed in anticipation of the necessary long-term hospitalization. During the 30-day hospital isolation, greetings and communication from his family made him feel better. His depression was mainly caused by the source of infection being in his hometown. In the hospital, he believed that his mild symptoms posed little risk for others. However, when some health workers kept a certain physical distance away from him in the ward, he felt that "he was being discriminated against", and thought that "they were isolating him deliberately". Consequently, he felt more depressed and angry about the discrimination. After he was discharged from the hospital, he was discriminated against by quite a few people in his hometown as they considered him to be "a potentially infectious and thus, dangerous patient". He complained of feeling like an outcast and even "doubted kinship and friendship". During this time, he told himself that he would control himself and not care about others' comments on him. He did not conceal his infection experience because he considered himself a "victim". He did not understand why other people would discriminate against recovered patients like him. Therefore, he was in a poor state of mind during that period. He was irritated by the frequent COVID tests required by the quarantine office. He even quarreled with health workers and blamed them for wasting 
national assets. This further increased his negative mood. He felt that "the medical workers had not stood in his shoes". However, he finally accepted the COVID tests during the next phase. A period of time later, his depression was assuaged because his friends and relatives gradually changed their attitude towards him.

\section{$6^{\text {th }}$ Case: "I did not want to have negative effects on others"}

The 34-year-old man (subject 6) was admitted to the hospital after testing positive for SARS-CoV-2. COVID19 caused a few mild symptoms such as fatigue. Initially, he felt tired easily and had flu-like symptoms. As reported by him, he was possibly infected by his relatives. At the beginning of hospital quarantine, he worried immensely that "he would not recover as there was no effective medicine or treatment for COVID-19". Hence, he was perturbed and anxious at this stage. With time going by, his anxiety was gradually relieved. 14 days later, he was discharged after testing negative. Later, he was encouraged to donate blood for research. Unfortunately, the nucleic acid test for blood donation showed that he was positive for SARS-CoV-2 again. He suffered from insomnia the first day he was re-admitted to the hospital. He had another 28 days of hospital quarantine until recovery. What was worse was that he retested positive for a third time only two weeks after the second discharge. During the second and third repositivity, he felt depressed because "the coronavirus had a negative effect on people around him". Although he did not talk about his infection with others, his diagnosis of COVID-19 was known by his friends and local people. His relatives and friends showed fear towards him and avoided greeting or meeting him. Psychologically, he worried about his social relationships. He did not want others to ignore him for fear of him. This made him more anxious and depressed. He worried about meeting his family as he did not want his family to be negatively affected by the repercussions of his re-positivity. As he recollected, he "did not sleep well when he felt anxious", but this did not last a long time in the hospital because his physical symptoms were relatively mild. The two re-positivity experiences had caused him to adjust his mood and attitude to despair.

\section{DISCUSSION}

In our case series, the six retest positivity COVID-19 patients showed various psychological problems such as anxiety, depression, excessive worries, irritation, low mood, stress, stigma, distress, grief, feelings of guilt, and loneliness (Chen et al. 2020, Luo et al. 2020, Sahoo et al. 2020a). Our results suggest that poor mental health is severely impacting the well-being of patients who experience repeated COVID-19 retest positivity results.

Our findings are consistent with the literature, in that mental health problems are known to be caused by quarantine given the social disconnectedness of quarantine, and perceptions of social isolation are known to be initiating factors for mental health issues such as anxiety, depression, and anger (Luo et al. 2020, Smith et al. 2020, Santini et al. 2020). In our data, subject 1 had thoughts of suicide after 47 days of hospital quarantine and 30 days of home quarantine. This is in line with Mei et al.'s (2020) findings regarding suicidal ideation among retest positivity patients. Subject 1's narrative metaphor "my brain broke down for constant imagination about the misfortune" reflects her extreme mental state concerning her disconnectedness from the outside world during the second infection. In the same way, extended periods of treatment and hotel quarantine made subject 2 irritated and extremely frustrated. She refused to go to the hospital after the second retest positivity result. The constant inquiries from friends and relatives increased her grief and stress. Subject 5 had 42 days of hospital quarantine, including 12 days during the first admission and 30 days during the second admission. The long period of quarantine led to severe symptoms of worry, anxiety, depression, anger, and irritation due to his perception of social isolation. He even quarreled with health staff. Taken together, these findings reveal that the experience of re-positivity resulted in an extended period of quarantine time which altered patients' perceptions of social connectedness.

In addition to social isolation, risk factors including perceived danger, uncertainty, physical discomfort, fear of infecting others, limited family support, and negative news in mass media coverage also lead to mental health issues such as loneliness, anger, anxiety, depression, insomnia, and post-traumatic stress symptoms (Bo et al. 2020, Luo et al. 2020, Sahoo et al. 2020a). Notably, factually vague information coverage in social networking platforms (e.g., WeChat and Weibo) and social discrimination may result in higher prevalence of selfperceived post-traumatic stress symptoms (Bo et al. 2020). In the present study, subject 1 trusted only her fellow patients due to extreme mental isolation; subject 2 encountered discrimination from neighbors; subject 5 mentioned that medical staff sometimes kept a distance with COVID-19 patients for fear of being infected; subject 6 worried about social relationship problems because his repeated positivity potentially exposed people around him to his infection. All these experiences of frustration changed patients' perception of social connectedness and negatively impacted on their social lives, occupational activities, and overall quality of life. Hence, enhancing social support systems (medical staff, family, friends, neighbors, and colleagues) and eliminating perceived stigma concerning COVID-19 are critical in reducing patients' psychological problems (Bao et al. 2020).

The present study has implications for potential therapeutic psychological interventions for COVID-19 patients who suffer from the fear of the disease itself as well as mental health problems such as loneliness, depression, irritation, anger, and anxiety for the welfare 
of family and friends. First, the uncertainty of having a deadly illness, limited family support, social discrimination, and fear of death show that mental health evaluation and mental health support to COVID-19 patients' needs to be routinely included as an essential component of the therapeutic workup (Sahoo et al. 2020a). For patients who suffer from post-discharge retest positivity, interventions should be dictated by a comprehensive assessment of risk factors leading to mental problems at different stages (Duan \& $\mathrm{Zhu}$ 2020, Xiang et al. 2020). During our interviews, the six patients with COVID-19 demonstrated severe symptoms of mental health disorder during the readmission period, suggesting that it is crucial to evaluate the psychological status of patients during both the period of hospitalization as well as the period of self-quarantine once the risk of re-positivity is confirmed. Apart from monitoring physical symptomatic reoccurrence, special cautions should be exercised in patients who suffered from both perceived stigma and social discrimination, in the sense that they are likely to be vulnerable under the oppressive conditions of guilt and discrimination. Second, mental health professionals and their clinical medical colleagues should collaborate in COVID-19 treatment centers to diagnose both the risk factors for symptomatic reoccurrence as well as the psychological needs of retest positivity patients to reduce their pain both physically and psychologically. In this regard, psychological management should become an integral part of COVID-19 risk assessment (Sahoo et al. 2020b). Third, patients' privacy should be protected in current clinical practice and post-COVID era mental health support. The repositivity patients in this study encountered different degrees of social discrimination from neighbors and friends due to exposure of their identities during readmission. As a result, they avoided social connection. For instance, the medical personnel's regular visiting and monitoring procedures on the symptomatic reccurrence of subjects 1 and 5 increased their pressure and irritation, in that they felt like being inspected by others even after recovery. During psychological interventions, mental health professionals and medical staff can provide counseling for COVID-19 patients by telephonic consults or online social media networking to protect patients' privacy (Sahoo et al. 2020b). Finally, social interactions among COVID-19 patients play an important role in reducing the emergence of mental health problems during the period of re-positivity. In our study, subject 1 only trusted the recovered COVID-19 patients as opposed to medical staff or family members. Subject 4 was optimistic during the treatment process with fellow patients. There is no doubt that patients undergoing similar medical adversities can understand and communicate effectively with each other during the recovery process. Therefore, the role of recovered fellow COVID-19 patients in therapeutic psychological interventions should not be underestimated.

\section{CONCLUSION}

This study demonstrated that post-discharge re-positivity COVID-19 patients experienced psychological problems such as fear, anxiety, depression, loneliness, anger, perceived stigma, worry, insomnia, and despair. The main source of mental health problems in these patients can be attributed to factors such as long quarantine times, small isolation space, fear of virus transmission to others, limited family support, concerns about delayed return to work, and negative news in mass media coverage (Epstein et al. 2020, Luo et al. 2020). In clinical practice, close monitoring of patients on an outpatient basis is crucial to assess the symptomatic recurrence of COVID-19, even after they have apparently overcome the initial infection (Lafaie et al. 2020, Ravioli et al. 2020). Equally important, routine evaluation by mental health professionals should be an integral part of current and future COVID-19 treatment protocols. Psychiatrists, medical staff, family members, and recovered patients can unite to engage and confront mental health consequences concerning the multiple waves of infection and reinfection with COVID-19 (Luo et al. 2020, Sahoo et al. 2020b).

\section{Acknowledgments:}

We would like to show gratitude to all of the interviewees who participated in this study voluntarily. The first author thanks his graduate students Quanquan Zhou, Xiaoyang Luo, Yang Liu, and Zhichun Duan for the assistance in the data processing.

\section{Funding:}

This study was supported by the Education Reform Project on Language Pathology, Sichuan International Studies University (yjsjg201705)

\section{Conflict of interest: None to declare.}

\section{Contribution of individual authors:}

Design: Yu Deng, Li Wang \& Yaokai Chen; Writing-original draft: Yu Deng; Writing-review and editing: Yaokai Chen; Visited the patient, collected the clinical data: Li Wang Investigation: Li Wang, Jixue Yang \& Luxue Xie; Supervision: Yaokai Chen.

\section{References}

1. Arafkas M, Khosrawipour T, Kocbach P, Zielinski K, Schubert J, Mikolajczyk A et al.: Current meta-analysis does not support the possibility of COVID-19 reinfections. J Med Virol 2021; 93:1599-1604

2. Bao Y, Sun Y, Meng S, Shi J \& Lu L: 2019-nCoV epidemic: address mental health care to empower society. Lancet 2020; 395:e37-e38

3. Bo H, Li W, Yang Y, Wang Y, Zhang $Q$, Cheung $T$ et al.: Posttraumatic stress symptoms and attitude toward crisis 
mental health services among clinically stable patients with COVID-19 in China. Psychol Med 2020; doi:10.1017/S0033291720000999

4. Bongiovanni M: COVID-19 re-infection in a healthcare worker. J Med Virol 2021. doi:10.1002/jmv.26565

5. Chen J, Tian C, Cheng X, Huang $Y$, \& Zeng X: A case of coronvirus disease 2019 with psychological disorders. Psychiatr Danub 2020; 32:581-583

6. Dong H, Hu R, Lu C, Huang D, Cui D, Huang $G \&$ Zhang M: Investigation on the mental health status of pregnant women in China during the Pandemic of COVID-19. Arch Gynecol and Obstet 2020; 2:303

7. Duan L\& Zhu G: Psychological interventions for people affected by the COVID-19 epidemic. Lancet Psychiat 2020; 7:300-302

8. Epstein D, Andrawis W, Lipsky AM, Ziad HA \& Matan M: Anxiety and suicidality in a hospitalized patient with COVID-19 infection. Eur J Case Rep Intern Med 2020; 7:1

9. Guessoum SB, Lachal J, Radjack R, Carretier E, Minassian S, Benoit L \& Moro MR: Adolescent psychiatric disorders during the COVID-19 pandemic and lockdown. Psychiatry Res 2020; 291: 113264

10. Islam MS, Ferdous MZ \& Potenza MN: Panic and generalized anxiety during the COVID-19 pandemic among Bangladeshi people: an online pilot survey early in the outbreak. J Affect Disord 2020; 276:30-37

11. Iwasaki A: What reinfections mean for COVID-19. Lancet Infect Dis 2020; 1:307830

12. Lafaie L, Celarier T, Goethals L, Pozzetto B, Grange S, Ojardias $E$ et al.: Recurrence or relapse of COVID-19 in older patients: a description of three cases. J Am Geriatr Soc 2020; 68:16728

13. Lee JS, Kim SY, Kim TS, Hong KH, Ryoo NH, Lee J et al.: Evidence of severe acute respiratory syndrome coronavirus 2 reinfection after recovery from mild coronavirus disease 2019. Clin Infect Dis 2020. doi:10.1093/cid/ciaal421

14. Luo X, Estill J, Wang Q, Lv M, Liu Y, Liu E \& Chen Y: The psychological impact of quarantine on coronavirus disease 2019 (covid-19). Psychiatry Res 2020; 291:113193

15. Mei Q, Li J, Du R, Yuan X, Li M \& Li J: Assessment of patients who tested positive for COVID-19 after recovery. Lancet Infect Dis 2020; 20:1004-1005
16. Muruganandam P, Neelamegam S, Menon V, Alexander $J$ \& Chaturvedi SK: COVID-19 and severe mental illness: impact on patients and its relation with their awareness about COVID-19. Psychiatry Res 2020; 291:113265

17. Parry J: Covid-19: Hong Kong scientists report first confirmed case of reinfection. BMJ 2020; 370:m3340

18. Prado-Vivar B, Becerra-Wong M, Guadalupe JJ, Márquez S, Gutierrez B, Rojas-Silva P et al.: A case of SARS-CoV-2 reinfection in Ecuador. Lancet Infect Dis 2020. doi:10.1016/S1473-3099(20)30910-5

19. Rajkumar RP: COVID-19 and mental health: a review of the existing literature. Asian J Psychiatr 2020; 52:102066

20. Ravioli S, Ochsner $H$ \& Lindner G: Reactivation of COVID-19 pneumonia: a report of two cases. J Infect 2020; 81:e72-e73

21. Sahoo $S$, Mehra A, Suri $V$, Malhotra $P \&$ Grover $S$ : Handling children in covid wards: a narrative experience and suggestions for providing psychological support. Asian J Psychiatr 2020a; 53:102207

22. Sahoo S, Mehra A, Suri V, Malhotra P, Yaddanapudi LN, Dutt Puri G \& Grover S: Lived experiences of the corona survivors (patients admitted in COVID wards): a narrative real-life documented summaries of internalized guilt, shame, stigma, anger. Asian J Psychiatr 2020b; 53:102187

23. Santini ZI, Jose PE, Cornwell EY, Koyanagi A, Nielsen L, Hinrichsen $C$ et al.: Social disconnectedness, perceived isolation, and symptoms of depression and anxiety among older Americans (NSHAP): a longitudinal mediation analysis. Lancet Public Health 2020; 5:e62-e70

24. Smith L, Jacob L, Yakkundi A, McDermott D, Armstrong NC, Barnett $Y$ et al.: Correlates of symptoms of anxiety and depression and mental wellbeing associated with COVID-19: a cross-sectional study of UK-based respondents. Psychiatry Res 2020; 291:113138

25. Wang C, Zhou J \& Zong C: Two cases report of epidemic stress disorder to novel coronavirus pneumonia. Asian $J$ Psychiatr 2020; 51:102070

26. Xiang YT, Yang Y, Li W, Zhang L, Zhang $Q$, Cheung $T \&$ $\mathrm{Ng} \mathrm{CH}$ : Timely mental health care for the 2019 novel coronavirus outbreak is urgently needed. Lancet Psychiat 2020; 7:228-229

Correspondence:

Professor Yaokai Chen, MD, PhD

Division of Infection Diseases, Chongqing Public Health Medical Center

109 Baoyu Road, Shapingba District, Chongqing, 400036, PR China

E-mail:yaokaichen@hotmail.com 